\title{
SENSORS AS AN ENABLER FOR SELF-OPTIMIZING GRINDING MACHINES
}

\author{
M. Maier ${ }^{1 *}$, T. Gittler ${ }^{1}$, L. Weiss ${ }^{1}$, C. Bobst ${ }^{2}$, S. Scholze ${ }^{2}$, K. Wegener $^{3}$ \\ ${ }^{1}$ inspire AG, Technoparkstrasse 1, 8005 Zürich, Switzerland \\ ${ }^{2}$ Agathon AG, Gurzelenstrasse 1, 4512 Bellach, Switzerland \\ ${ }^{3}$ ETH Zürich, Institute of Machine Tools and Manufacturing (IWF), 8092 Zürich, Switzerland \\ ${ }^{*}$ Corresponding author; e-mail: maier@inspire.ethz.ch
}

\begin{abstract}
Today, an operator performs experiments to adaptively select grinding process parameters using observations, expert knowledge, and rules of thumb. Self-optimizing grinding machines cannot use operator observations and must therefore extract enough information out of the grinding process. In this study, a holistic sensor set-up as foundation for self-optimizing machines are presented exemplarily for cup wheel grinding machines. In-process detection of grinding burn, based on temperature and gas measurements, is tested and compared. Afterwards, the influence of input variables such as feed rate and cutting speed on grinding cost, grinding burn, and surface roughness are investigated.
\end{abstract}

\section{Keywords:}

Sensor fusion; Self-optimizing machines; Cost calculation; Process boundary; Grinding burn; Gas sensor; Temperature sensor; Surface roughness

\section{INTRODUCTION}

Most often, grinding is one of the last of several manufacturing steps and non-optimized machines or workpiece quality defects lead to high productivity losses. Different approaches for the optimization of grinding processes exist. [Venkata Rao 2011] presented an overview of grinding optimization, using different models and optimization techniques. For example, [Choi 2007] showed the optimization of a grinding process by using a combination of empirical and physical models. This approach works well for situations where extensive training data is available or low model complexity is sufficient. However, this approach may fail or needs recalibration for situations outside the trained scope of the optimization, such as new workpiece, tool, and machine combinations.

Another approach was taken by [Morgan 2007], where a combination of sensor data and rules is used to optimize the grinding process. The usage of sensor data is an advantage because it provides feedback to the optimization system. In this way, the optimization can react to the observed data. A disadvantage is that decisions based on rules restrict the flexibility of the system. Many rules are a result of compromises because the grinding operation is too complex to foresee every possible grinding situation, which leads to non-optimal processes.

The ingredients for a self-optimizing grinding machine are sensors, model/algorithm and database. This is similar to the approach of today's operators. The operators use knowledge from previous experiments, which are comparable to the database of self-optimizing machines, acoustic and olfactory observations which are comparable to sensors, and rules of thumb which represent simple models of specific aspects of the grinding process.
Recently, a new approach for self-optimizing machines was tested for turning [Maier 2019a], [Maier 2019b]. It is composed of Bayesian optimization, a sequential design strategy [Shahriari 2016], combined with a stochastic Gaussian process model [Rasmussen 2006] for the autonomous process set-up in turning. The method allows for the optimization of new grinding tasks, such as new workpiece, tool and machine combinations, with only a very limited number of experiments. Fig. 1 shows a schematic concept for Bayesian optimization in grinding. Bayesian optimization allows to determine the relation of the input variables towards an output, where an objective, such as a cost or utility, is minimized or maximized. In general, the optimization objective includes various aspects such as production costs, energy supplied to the machine tool, and environmental impact. In this study, the objective was to minimize production costs, which mainly consist of machining time costs and tool wear costs. The optimization might be subject to constraints, such as input variables and quality constraints. Input variable constraints such as maximal or minimal feed rates are known a priori. They can easily be implemented in the optimization by specifying the optimization input domain accordingly. In grinding, quality constraints might be imposed by surface roughness and/or workpiece surface integrity requirements. Surface roughness and surface integrity are constraint properties of the workpiece, which must be modelled. Based on available cost and constraint value measurements, stochastic process models are used to predict cost and constraint values and corresponding uncertainties for each input variable combination. The predicted cost and constraint values are then used to decide where the next experiment shall be conducted. The Bayesian optimization algorithm trades off experiments with high predictive value 
(exploitation) and high predicted uncertainties (exploration). This process is repeated until a given number of iterations or a convergence criterion is fulfilled. Mathematically, the algorithm optimizes problems of the following form,

$$
\boldsymbol{x}_{\boldsymbol{o p t}}=\operatorname{argmin} C_{T}(\boldsymbol{x}) \text { s.t. } \boldsymbol{c}(\boldsymbol{x})<\boldsymbol{c}_{\max }
$$

where $\boldsymbol{x}_{\boldsymbol{o p t}}$ are the optimal input variables, $\boldsymbol{x}$ are the input variables, $C_{T}$ are the total production costs, and $\boldsymbol{c}$ are constraint values constrained to the maximum allowed constraints $c_{\max }$.

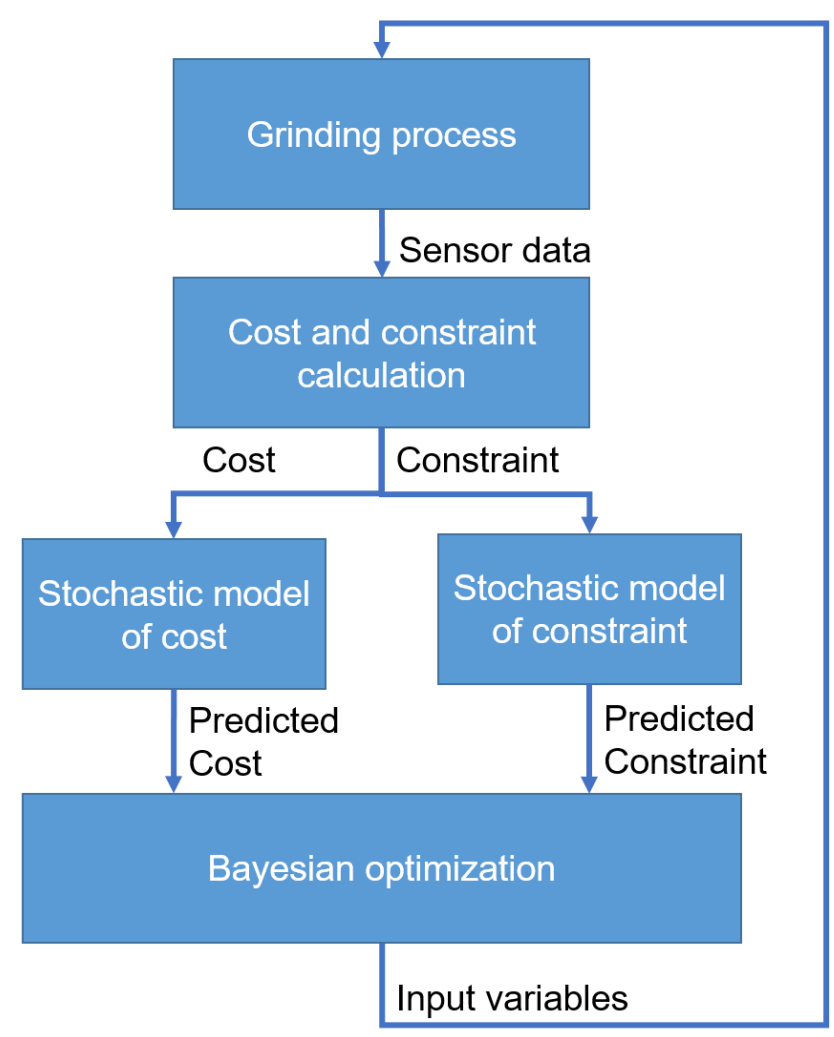

Fig. 1: Schematic concept of Bayesian optimization in grinding.

In this study, information extraction for the optimization of cup wheel grinding of tungsten carbide inserts is investigated exemplarily. The paper focuses on the dedicated combination of sensors for cost and constraint calculation as a first step for successive optimization. Results from gas and temperature sensor measurements for in-process grinding burn detection are presented and compared. The results are discussed in the context of a sensor fusion approach, comparing the system's output with results from current literature, as well as the results currently obtained on the examined machines via purely operator knowledge-based process setups.

\section{SENSORS FOR COST AND CONSTRAINT MEASUREMENT}

For the use of new optimization techniques in grinding, such as proposed in Fig. 1, new sensors are needed. The sensor data must be able to measure the process adherence to cost and constraint values for each input variable combination. Fig. 2 summarizes some typical input variables, measured cost quantities, cost parameters, process parameter and workpiece quality constraints for calculation of cost and constraint values in cup wheel grinding of cutting inserts. Input variables are tuned to accomplish a predefined grinding task. In grinding, several input variables must be tuned, such as feed rate and cutting speed, but also the grinding and dressing wheel type might vary. Total production cost consists of measured quantities, such as dressing time, maximum dressing interval and grinding time, as well as cost parameters, such as machine hourly costs and costs of grinding wheel. The cost parameters are not measured during grinding - these parameters result from factory data capture. Cost parameters depend on the location of the production site, purchasing contracts and/or workload of machines. Some measured quantities such as the grinding time can be calculated in a straightforward manner. The grinding time depends directly on removed workpiece material and feed rate of the grinding operation. The maximum dressing interval may be determined by grinding burn measurements. The measured costs can also include replacement costs for utilities caused by grinding and dressing wheel wear. The inputs to the total costs and constraints are mainly determined by the grinding application and the desired granularity of the calculation. The cup wheel grinding process is mainly bound by workpiece quality constraints. Today, dimensional accuracy is controlled by the machine with an in-process measurement probe and a corrective feedback control loop. Workpiece surface roughness can be measured by tactile or optical measurement devices as shown by [Azarhoushang 2017] for optical surface roughness measurements in cup wheel grinding of inserts. One major challenge of the cup wheel grinding process is the detection of grinding burn, which causes severe damage of the workpiece. Grinding burn does not only limit the process, it also determines the maximum dressing interval. Grinding temperature strongly depends on the grinding kinematics [Malkin 2008]. Grinding burn of the final workpiece can be detected by optical inspection, nital etching [Mayer 1999], Barkhausen noise analysis [Karpuschewski 2011; Thanedar 2017], X-ray diffractometry [Sinha 2016], or by using a Hall probe [Teixeira 2019]. Instead of directly measuring cost quantities and quality constraint values, process parameters such as force, power, and temperature can be used to infer on these values. In an industrial environment, operators also detect grinding burn during operation indirectly by burn of grinding lubricant, which can be detected olfactorily. Tab. 1 summarizes industrial and lab sensors for process parameters in grinding and their applications. An extensive review for process monitoring in grinding is given by [Tönshoff 2002]. Several methods for grinding burn detection exist. Using temperature measurements of the grinding zone directly is very promising for grinding burn detection as it allows to directly measure the phenomenon itself, without having to revert to observations or inference. [Jermolajev 2014] show grinding burn detection of cylindrical grinding using grinding temperature measurements at the contact zone. 


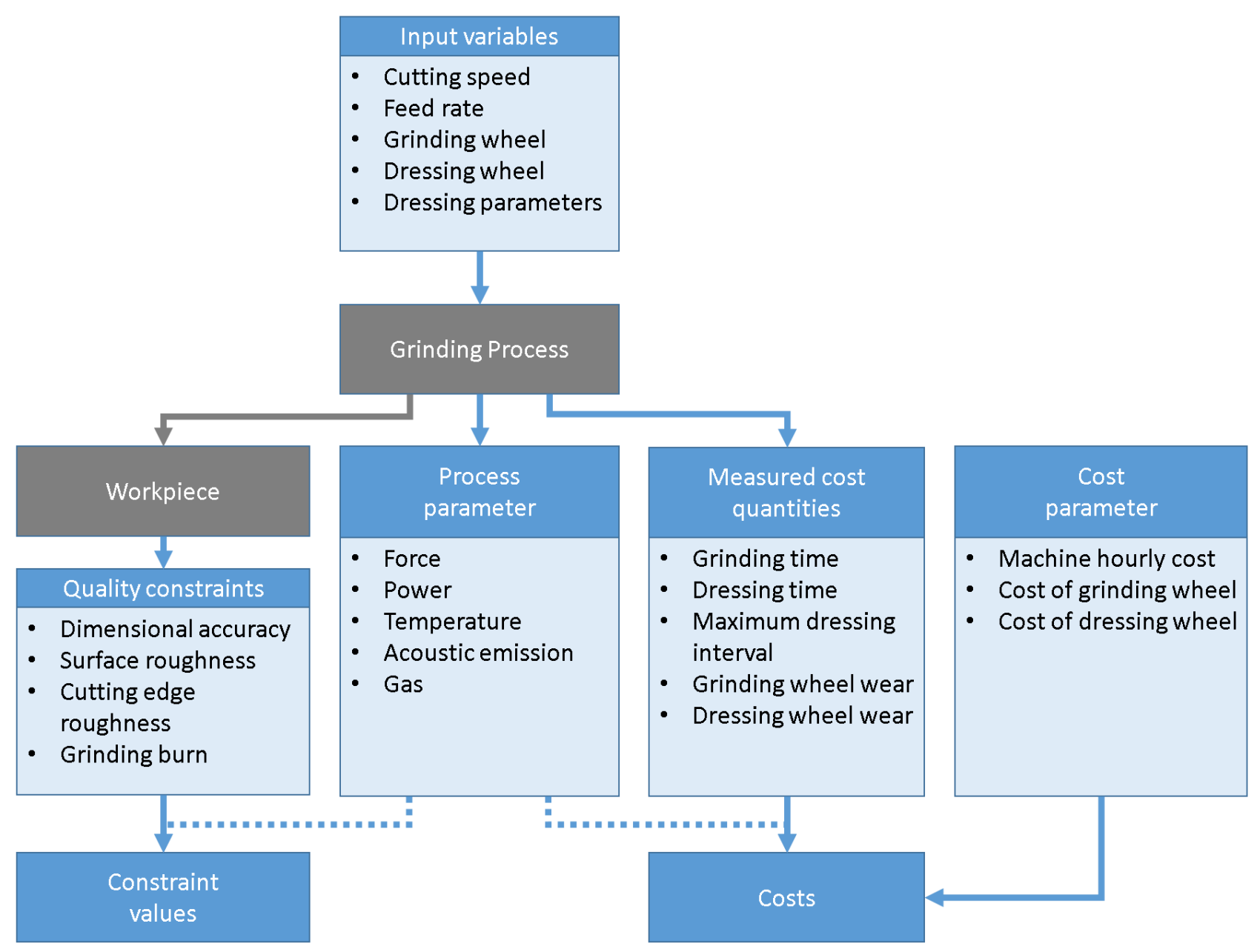

Fig. 2: Input variables, measure cost quantities, cost parameter, process parameter, and workpiece quality constraints of cup wheel grinding of inserts for calculation of cost and constraint values.

Tab. 1: Sensors for process monitoring and their applications in grinding.

\begin{tabular}{lll}
\hline Reference & Measured quantity & Application \\
\hline [Tönshoff 1986] & Force & Grinding contact detection \& force controlled grinding \\
[Govekar 2002] & Force & Chatter detection \\
[Morgan 2007] & Power & Spark-out time reduction \\
[Inasaki 1991] & Power & Burn detection \& Grinding wheel life detection \\
[Jermolajev 2014] & Temperature & Prediction of workpiece surface layer properties \\
[Karpuschewski 2000] & Acoustic emission & Dressing monitoring \\
[Lange 2016] & Acoustic emission & Detection of run-out error \\
[Yang 2012] & Acoustic emission & Classification of sharp and dull grinding wheels \\
[Yang 2014] & Acoustic emission & Grinding burn detection \\
\hline
\end{tabular}

To the authors knowledge, grinding burn in cup wheel grinding of inserts using temperature measurements has not been studied specifically. No study has been found using gas sensor measurements for burn detection in grinding. Grinding burn detection using gas sensors is very attractive because investment costs are low.

Several studies investigated cup wheel grinding of inserts e.g. [Azarhoushang 2017] studied normal and tangential forces, surface roughness, and wheel loading, [Denkena 2014] investigated wheel topography, maximum edge chipping, surface roughness, and grinding forces, [Denkena 2015] studied grinding wheel wear, surface roughness, and cutting edge chipping. These studies investigated specific cost and constraint values. A comprehensive sensor set-up for optimization is missing. In this study, a holistic measurement set-up is presented, which can be used as a foundation for self-optimizing cup wheel grinding machines. 


\section{METHODOLOGY}

\subsection{Grinding operation}

Fig. 3 shows a picture of the used cup wheel grinding machine (Agathon DOM). The machine is equipped with a metal-bonded diamond grinding wheel (Tyrolit D46C100M717). The grinding wheel rotates in counterclockwise direction. The rotational speed and the diameter of the grinding wheel specify the cutting speed. The grinding wheel can be moved in X-direction, which is the direction of the feed rate during grinding. To ensure a uniform wear of the grinding wheel, the grinding wheel is oscillated during grinding along the $\mathrm{Y}$-direction. The hard metal-bonded grinding wheel shows a limited selfsharpening effect. Therefore, a dressing wheel (Tyrolit 89A $240 \mathrm{~J} 5 \mathrm{AV} 217$ ) is used for conditioning. The workpiece is clamped using a clamping device, which can rotate the workpiece along the $\mathrm{B}$ axis. An initially quadratic insert ( $14.5 \mathrm{~mm} \times 14.5 \mathrm{~mm} \times 4.76 \mathrm{~mm}$ ) made of tungsten carbide (Tribo S25, grain size $2.5 \mu \mathrm{m}, \mathrm{HV} 301470$ ) is ground on two opposite sides. On each side $2.25 \mathrm{~mm}$ of workpiece material is removed. Resulting in a final workpiece geometry of $10 \mathrm{~mm} \times 14.5 \mathrm{~mm} \times 4.76 \mathrm{~mm}$. The cooling lubricant Blasogrind HC 5 (from Blaser Swisslube) is used during grinding and conditioning at a constant flow rate.

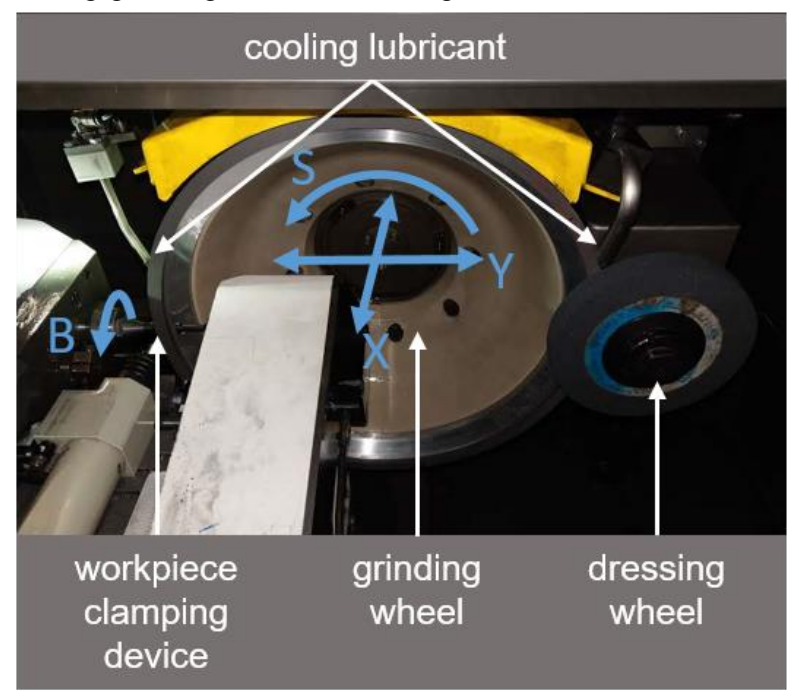

Fig. 3: Overview of cup wheel grinding machine.

\subsection{Cost calculation \& grinding burn}

In a very simplified form, the costs to grind one insert can be calculated as follows,

$$
C_{T}=C_{M} \frac{s \cdot a}{f}+\frac{C_{D}}{i_{d}}
$$

where $C_{M}$ is the machine hourly costs, $s$ is the number of ground sides per insert, $a$ is the removed material per side, $f$ is the feed rate, $C_{D}$ is the dressing costs, and $i_{d}$ is the dressing interval. The dressing interval is the number of parts ground between two dressing cycles. Idle costs are neglected as their consideration would merely result in a shift of the curve by a constant value. The machine hourly costs are assumed to be $100 \mathrm{U} / \mathrm{h}$ and the dressing costs are assumed to be $0.6 \mathrm{U}$ per dressing. The costs are specified in Swiss francs, but for generality, the cost unit is denoted in arbitrary units $U$. The machine hourly costs and the dressing costs can be interpreted as weighting factors for the total production costs. The cutting time can be directly calculated by the feed rate and the removed material per insert. The dressing interval is unknown a priori and must be measured experimentally.

The maximum dressing interval is measured by grinding with a freshly dressed wheel until grinding burn occurs. In this study, grinding burn is inspected optically after grinding. Workpieces with grinding burn show a distinct black coloring of the final workpiece surface (see Fig. 4). This approach is a simple approach for burn detection, which is widely used in industry. The main disadvantages of the method are that it is a subjective measurement and grinding burn below the surface may not be detected. Other methods for grinding burn detection exist, as discussed in section 2. However, the disadvantage of nital etching is that it is a subjective measurement such as the optical inspection and the disadvantage of the more sophisticated Barkhausen noise measurements is that it needs calibration and referencing [Karpuschewski 2011]. The simple inspection method is considered sufficient, as the aim of this study is to investigate a holistic sensor set-up for selfoptimizing grinding machines. In case of higher surface quality requirements, a more sophisticated method is recommended.

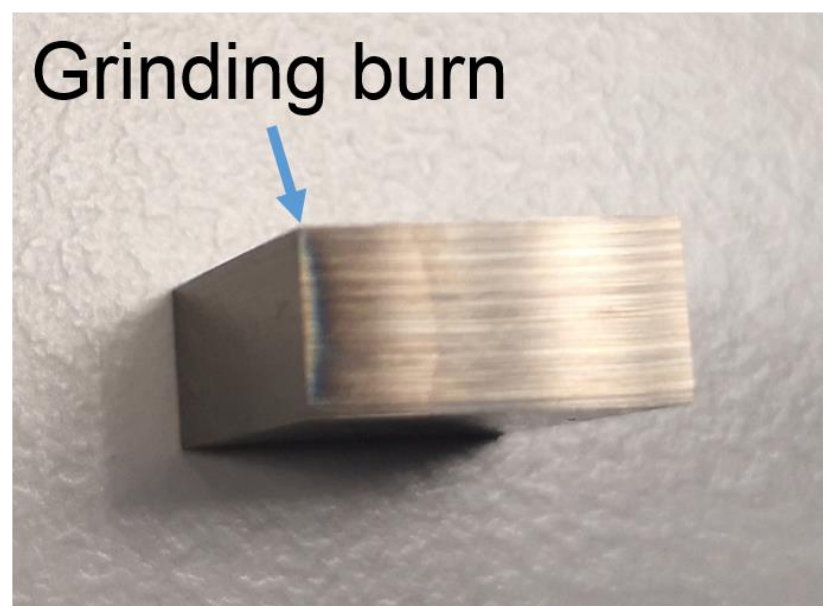

Fig. 4: Optical detection of grinding burn.

\subsection{Temperature measurement}

The grinding temperature is measured at the contact zone between grinding wheel and workpiece by use of an optical measurement system (FOS Messtechnik). The optical measurement system consists of four evenly distributed fibers which are embedded in the grinding wheel with an orientation perpendicular to the abrasive layer surface. The temperature signal is processed on the rotating cup wheel and transmitted wirelessly to a receiver unit outside the grinding machine. The receiver unit is connected to a measurement computer, where the data is post-processed. Fig. 5 illustrates the temperature measurement setup. The measurement system has a measurement range between 200 and $660^{\circ} \mathrm{C}$. Note that the emissivity of the workpiece is not measured. Therefore, the temperature readings can only be considered as relative measurements. However, a relative measurement is sufficient in this study because the final objective is to determine a threshold temperature for grinding burn detection. 


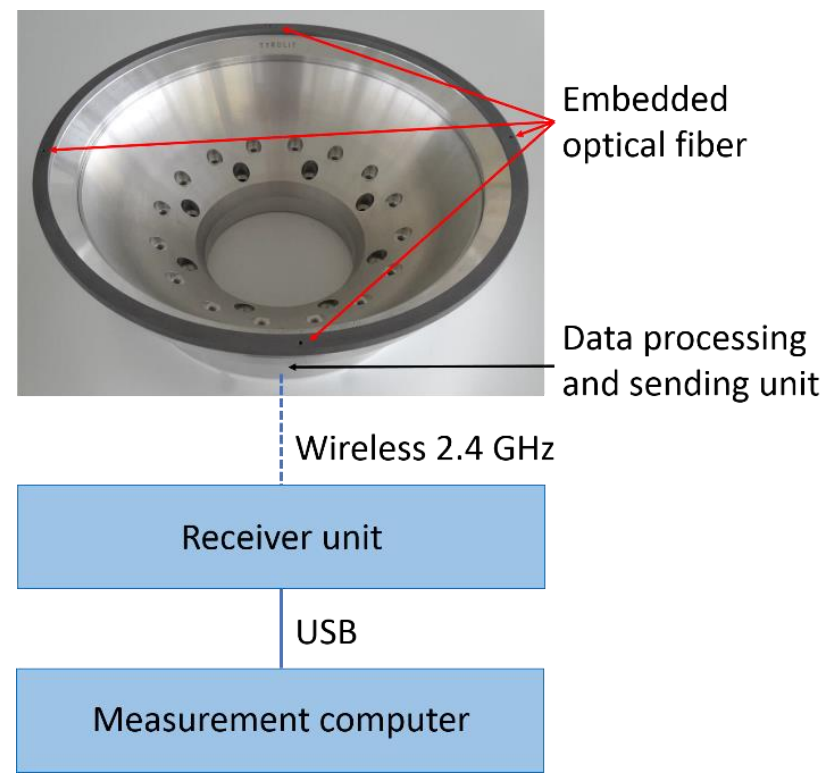

Fig. 5: Experimental setup of temperature measurement.

\subsection{Gas measurement}

The gas concentration is measured using a metal-oxide gas sensor (Sensirion SGP30). The sensor is able to detect total volatile organic compounds (TVOC) and can calculate a $\mathrm{CO}_{2}$ equivalent, which corresponds to the greenhouse gas emissions of the test gas. Originally, the sensor is designed for indoor air quality measurements. To protect the sensor from cooling lubricant, it is placed in a 3D-printed housing as shown in Fig. 6 . The housing is placed vertically inside the grinding machine. A ventilator inside the housing is used to provide a steady circulation of the ambient process chamber gases. The gas enters the housing on the bottom and is guided through plates as fluid separators to the gas sensor. Afterwards, the gas leaves the housing through a $U$-shaped tube to protect the sensor from cooling fluid.

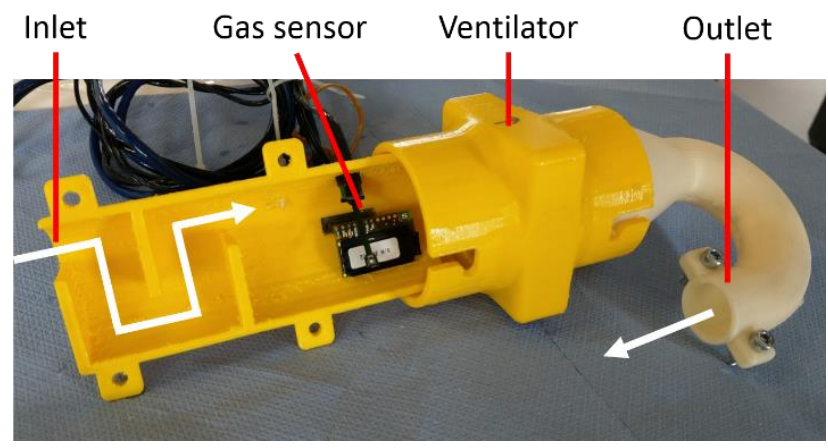

Fig. 6: Experimental setup of a gas sensor.

\subsection{Surface roughness measurement}

The surface roughness of the ground workpiece is measured transversally to the grinding direction using a tactile measurement devise (Taylor Hobsen Form Talysurf Series 2) with a tip radius of the measurement probe of $2 \mu \mathrm{m}$ and a cut-off wavelength of $\lambda_{c}=0.8 \mathrm{~mm}$. The measurement length is reduced to $3.5 \mathrm{~mm}$, since the total length of the workpiece was only $4.76 \mathrm{~mm}$. The roughness was measured on both ground sides of the insert and subsequently averaged.

\section{RESULTS}

\subsection{In-process detection of grinding burn}

Fig. 7 shows a comparison of in-process grinding burn detection based on maximum temperature measurements and on maximum gas concentration measurements. The experiments where conducted for different cutting speeds, feed rates, and wheel wear states of the grinding wheel. The temperature sensor is able to distinguish grinding burn and no grinding burn with a $100 \%$ success rate. No grinding burn was observed for maximum temperature readings below $585^{\circ} \mathrm{C}$. All temperature readings above the threshold value showed grinding burn. For process optimization, the measured temperature can be used as a constraint value and the maximum allowed temperature is determined to be $585^{\circ} \mathrm{C}$ (compare with equation (1)).

The maximum gas concentrations are correlated with the maximum grinding temperatures. An increase in maximum temperature leads to an increase in maximum gas concentration. The sharp peak at high temperatures is caused by the measurement range of the temperature sensor, which is limited to $660^{\circ} \mathrm{C}$. For the gas measurement, no clear threshold limit can be determined. A threshold limit of 2000 ppm detects all workpiece grinding burns but is a very conservative approach with respect to the grinding burn limit. A threshold of $6000 \mathrm{ppm}$ is able to detect cases without wrongly classifying successful grinding operations as failures. A threshold limit of $6000 \mathrm{ppm}$ can be seen as a limit for very extreme cases. The gas sensor is a low cost alternative for grinding burn detection with reduced sensitivity.

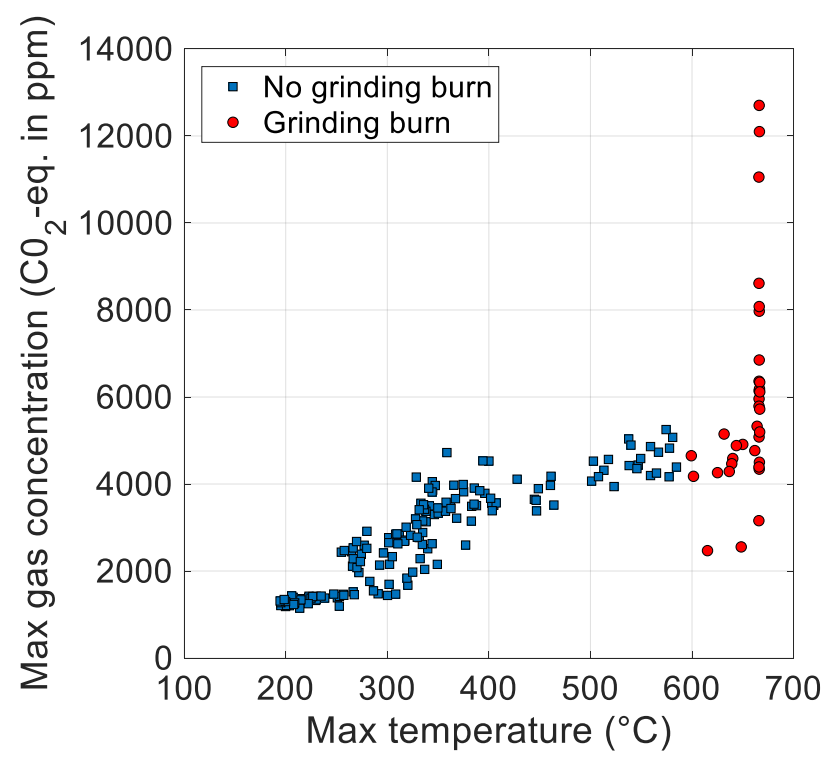

Fig. 7: Comparison between measured maximum gas concentration and maximum temperature for in-process detection of grinding burn.

\subsection{Grinding burn and surface roughness constraint}

Fig. 8 shows the influence of feed rate and cutting speed on grinding burn for grinding the first side of the test insert directly after dressing. Grinding burn is favored by high feed rates and high cutting speeds. In general, a higher uncut chip thickness reduces the specific grinding energy (energy required to remove a unit volume of material) [Rowe 2014]. According to [Malkin 2008] the uncut chip thickness is increased by increasing the feed rate but is reduced when the number of active grains is increased. However, an increase in feed rate simultaneously increases the number of active grains due to wheel flexibility [Rowe 2014]. 
Therefore, the effect of feed rate on specific energy is unknown a priori. [Azarhoushang 2017] reports that an increase in feed rate leads to an increase of specific energy in cup wheel grinding of PCBN cutting inserts, which indicates that for their test setup the influence of increase of active grains was dominate. Despite the contrary effects of feed rate on the specific grinding energy, high feed rates lead to a higher material removal rate, which increases the power demand and total heat dissipation. Higher cutting speeds increase the specific energy due to a decrease in uncut chip thickness, which reduces the energy efficiency of the process, and results in higher temperatures [Rowe 2014].

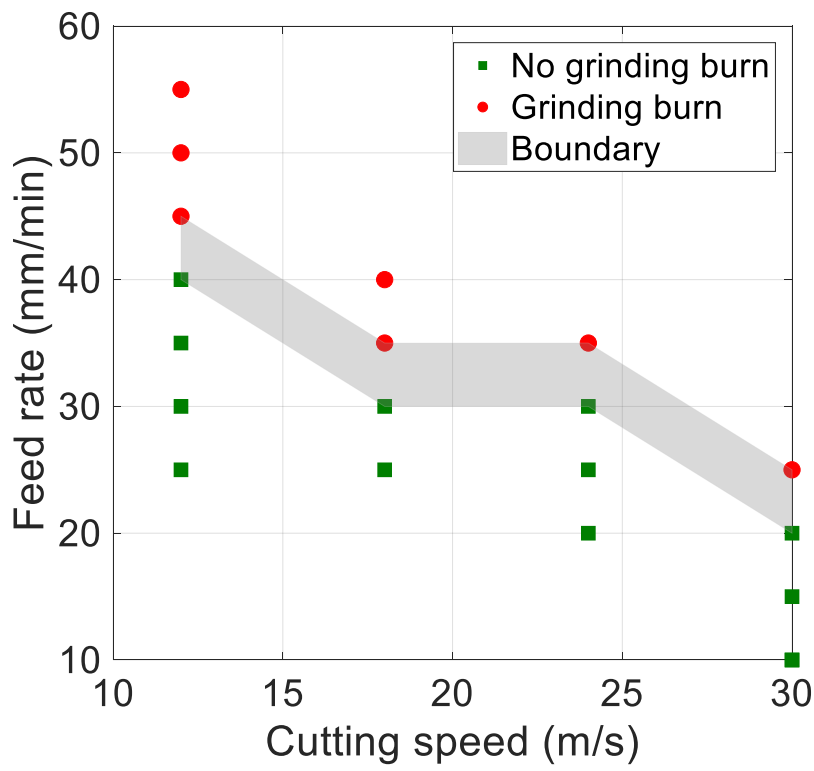

Fig. 8: Measured grinding burn limit of a freshly dressed cup wheel grinding process.

Fig. 9 shows the workpiece surface roughness as a function of cutting speed and feed rates. An increase in cutting speed leads to a decrease in surface roughness of the workpiece. This is in line with the findings of [Azarhoushang 2017]. For the feed rate no general trend can be observed.

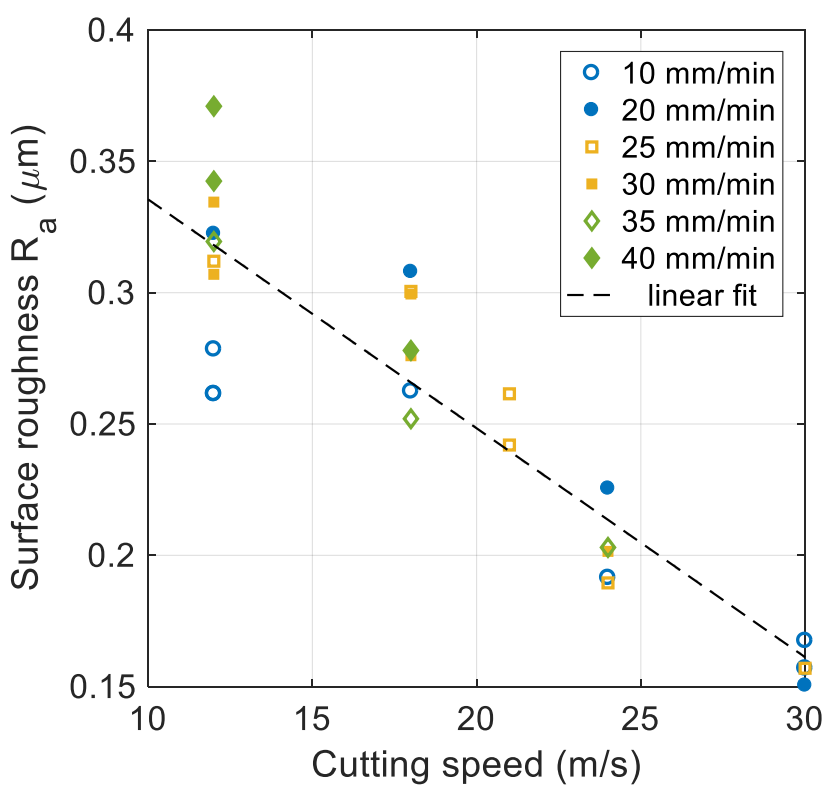

Fig. 9: Surface roughness of workpiece ground with a freshly dressed wheel for different feed rates and cutting

\subsection{Grinding cost}

Grinding costs are influenced by maximum dressing intervals. To measure the maximum dressing interval, a freshly dressed wheel is used for grinding until grinding burn occurred. The maximum number of ground inserts until grinding burn occurred is used for comparison, which can be seen in Fig. 10. After eight inserts, the experiment was stopped to avoid unnecessarily long experiments. An increase in cutting speed reduces the maximum number of ground inserts before exceeding the temperature limit drastically. An explanation for this effect is a decrease in the effectiveness of the self-sharpening effect at higher cutting speeds. An increase in feed rate leads to a reduced number of maximum ground inserts. For higher feed rate the temperature limit is reached sooner because the grinding operation is initially started at higher temperatures.

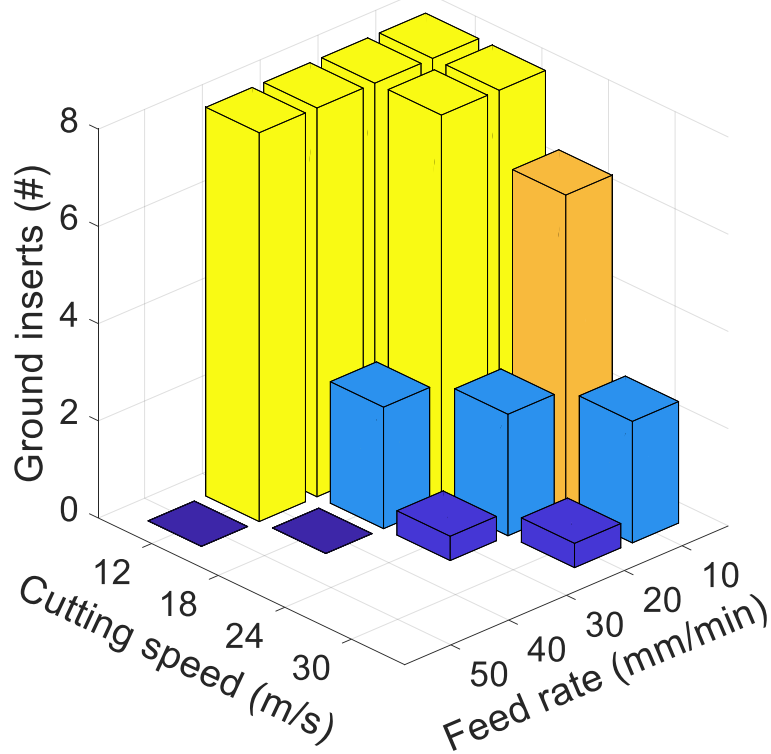

Fig. 10: Maximum dressing interval until temperature exceeds maximum.

Fig. 11 shows the grinding costs as a function of cutting speed and feed rate, calculated using equation (2). A higher cutting speed increases the grinding costs because higher cutting speeds result in shorter dressing intervals. The feed rate influences the grinding costs in two ways: On one hand, a higher feed rate reduces the grinding time, which leads to lower costs. On the other hand, high feed rates lead in general to shorter dressing cycles, which increases the dressing costs. These contrary effects determine the total costs. The lowest costs were achieved by grinding with low cutting speed and high feed rate. However, the surface roughness is high for low cutting speeds. Therefore, higher requirements of the final workpiece surface roughness result in optimal parameters with higher cutting speeds. For intermediate cutting speeds it is interesting to note that feed rates very close to the grinding burn limit lead to high costs. For these cases, the grinding operation should not be performed close to the grinding burn limit. 


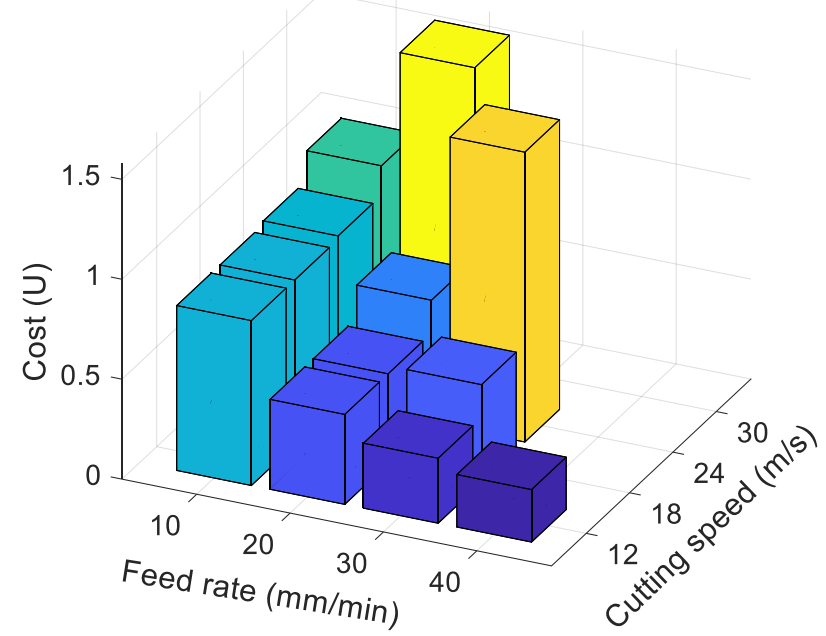

Fig. 11: Calculated costs using equation (2) as a function of feed rate and cutting speed.

\section{CONCLUSION}

In this study, a holistic approach for sensor-based information extraction for self-optimizing machines was investigated exemplarily for cup wheel grinding. Measuring production costs and quality constraints are essential for successive optimization. Grinding costs are influenced by different quantities, such as process time, dressing interval, dressing cost, and machine hourly costs. In this study's setting, the cost is minimal for low cutting speeds and high feed rates. Quality constraints come from surface roughness and grinding burn. Grinding burn is observed for high feed rates and high cutting speed. Surface roughness is reduced for high cutting speeds. Considering surface roughness requirements, the optimal cutting speed might be increased. In-process detecting of grinding burn is of high importance, as it is both an optimization constraint as well as an influence on the costs of the grinding operation. The optical temperature measurement system, integrated in the grinding wheel, shows excellent accuracy and reliable measurements. The system was able to detect grinding burn with a $100 \%$ success rate without false positives using a threshold temperature of $585^{\circ} \mathrm{C}$. The gas sensor is a low cost alternative with a reduced sensitivity.

A comprehensive sensor set-up was successfully demonstrated that can be used for constrained optimization. The sensor set-up is a foundation for selfoptimizing grinding machines. In future, the sensor set-up must be combined with optimization methods, such as Bayesian optimization, and a database.

\section{ACKNOWLEDGMENTS}

The project was funded by CTI number 25411. We thank T. Spiegelburg for the design and manufacturing of the gas sensor housing and S. Schmid for performing the surface roughness measurements.

\section{REFERENCES}

[Azarhoushang 2017] Azarhoushang, B., et al. Effects of grinding process parameters on the surface topography of PCBN cutting inserts. International Journal of Abrasive Technology, 2017, Vol.8, No.2, pp 121-132, ISSN 17522641
[Choi 2007] Choi, T. and Shin, Y. C. Generalized Intelligent Grinding Advisory System. International Journal of Production Research, 2007, Vol.45, No.8, pp 1899-1932, ISSN 0020-7543

[Denkena 2015] Denkena, B., Grove, T. and Behrens, L. Significant influence factors on the grinding tool wear and cutting mechanisms during grinding of PCBN inserts. Production Engineering, 2015, Vol.9, No.2, pp 187-193, ISSN 1863-7353

[Denkena 2014] Denkena, B., Köhler, J. and Ventura, C. Influence of grinding parameters on the quality of high content PCBN cutting inserts. Journal of Materials Processing Technology, 2014, Vol.214, No.2, pp 276-284, ISSN 0924-0136

[Govekar 2002] Govekar, E., et al. A New Method for Chatter Detection in Grinding. CIRP Annals, 2002, Vol.51, No.1, pp 267-270, ISSN 0007-8506

[Inasaki 1991] Inasaki, I. Monitoring and Optimization of Internal Grinding Process. CIRP Annals, 1991, Vol.40, No.1, pp 359-362, ISSN 0007-8506

[Jermolajev 2014] Jermolajev, S. and Brinksmeier, E. A New Approach for the Prediction of Surface and Subsurface Properties after Grinding. Advanced Materials Research, 2014, Vol.1018, pp 189-196, ISSN 1662-8985

[Karpuschewski 2011] Karpuschewski, B., Bleicher, O. and Beutner, M. Surface Integrity Inspection on Gears Using Barkhausen Noise Analysis. Procedia Engineering, 2011, Vol.19, pp 162-171, ISSN 1877-7058

[Karpuschewski 2000] Karpuschewski, B., Wehmeier, M. and Inasaki, I. Grinding Monitoring System Based on Power and Acoustic Emission Sensors. CIRP Annals, 2000, Vol.49, No.1, pp 235-240, ISSN 0007-8506

[Lange 2016] Lange, D. and Scherer, W. (2016) Mess- und Überwachungssysteme beim Schleifen Schweizer SchleifSymposium. Schweizer Schleif-Symposium. Zürich.

[Maier 2019a] Maier, M., et al. (2019a) Turning: Autonomous Process Set-up through Bayesian Optimization and Gaussian Process Models (in press). CIRP ICME 2019

[Maier 2019b] Maier, M., et al. Bayesian Optimization for Autonmous Process Set-up in Turning (in press). CIRP Journal of Manufacturing Science and Technology, 2019b, $\mathrm{pp}$, ISSN

[Malkin 2008] Malkin, S. and Guo, C. Grinding technology : theory and application of machining with abrasives. New York : Industrial Press, 2008.

[Mayer 1999] Mayer, J. E., Purushothaman, G. and Gopalakrishnan, S. Model of Grinding Thermal Damage for Precision Gear Materials. CIRP Annals, 1999, Vol.48, No.1, pp 251-254, ISSN 0007-8506

[Morgan 2007] Morgan, M. N., et al. Design and implementation of an intelligent grinding assistant system. International Journal of Abrasive Technology, 2007, Vol.1, No.1, pp 106-135, ISSN 1752-2641

[Rasmussen 2006] Rasmussen, C. E. Gaussian processes for machine learning. Cambridge, Mass: Cambridge, Mass : MIT Press, 2006.

[Rowe 2014] Rowe, W. B. Principles of modern grinding technology. Waltham, MA : William Andrew, 2014. 
[Shahriari 2016] Shahriari, B., et al. Taking the Human Out of the Loop: A Review of Bayesian Optimization. Proceedings of the IEEE, 2016, Vol.104, No.1, pp 148-175, ISSN 0018-9219

[Sinha 2016] Sinha, M. K., et al. An investigation on surface burn during grinding of Inconel 718. Journal of Manufacturing Processes, 2016, Vol.21, pp 124-133, ISSN $1526-6125$

[Teixeira 2019] Teixeira, P. H. O., et al. Application of Hall effect for assessing grinding thermal damage. Journal of Materials Processing Technology, 2019, Vol.270, pp 356364, ISSN 0924-0136

[Thanedar 2017] Thanedar, A., et al. Surface integrity investigation including grinding burns using barkhausen noise (BNA). Journal of Manufacturing Processes, 2017, Vol.30, pp 226-240, ISSN 1526-6125

[Tönshoff 2002] Tönshoff, H., Friemuth, T. and Becker, J. C. Process monitoring in grinding. CIRP AnnalsManufacturing Technology, 2002, Vol.51, No.2, pp 551571, ISSN 0007-8506

[Tönshoff 1986] Tönshoff, H. K., Zinngrebe, M. and Kemmerling, M. Optimization of Internal Grinding by Microcomputer-Based Force Control. CIRP Annals, 1986, Vol.35, No.1, pp 293-296, ISSN 0007-8506

[Venkata Rao 2011] Venkata Rao, R. Modeling and Optimization of Machining Processes. In: Advanced Modeling and Optimization of Manufacturing Processes: International Research and Development London: Springer London, 2011.

[Yang 2012] Yang, Z. and Yu, Z. Grinding wheel wear monitoring based on wavelet analysis and support vector machine. The International Journal of Advanced Manufacturing Technology, 2012, Vol.62, No.1, pp $107-$ 121, ISSN 1433-3015

[Yang 2014] Yang, Z., et al. Application of Hilbert-Huang Transform to acoustic emission signal for burn feature extraction in surface grinding process. Measurement, 2014, Vol.47, pp 14-21, ISSN 0263-2241 